\title{
The synergic effect of atomic hydrogen and catalyst spreading on Ge nanowire growth orientation and kinking
}

\author{
Miroslav Kolíbal ${ }^{1,2}$, Tomáš Pejchal ${ }^{2}$, Libor Novák ${ }^{3}$, Tomáš Vystavěl ${ }^{3}$, and Tomáš Šikola ${ }^{1,2}$ \\ ${ }^{1}$ Institute of Physical Engineering, Brno University of Technology, Technická 2, 61669 Brno, Czech \\ Republic \\ ${ }^{2}$ CEITEC BUT, Brno University of Technology, Technická 10, 61669 Brno, Czech Republic \\ ${ }^{3}$ FEI Company, Vlastimila Pecha 12, 62700 Brno, Czech Republic
}

In nanowire-based three-dimensional device architecture (e.g. gate-all-around nanowire field effect transistors) it is required to intentionally grow nanowires at precisely determined location and, additionally, perpendicular to the substrate. The former requirement was successfully met utilizing the so-called Vapor-Liquid-Solid (VLS) mechanism, where a eutectic catalyst droplet is used to localize the semiconductor material nucleation and growth to the droplet-substrate (liquid-solid) interface. The growth direction of VLS-grown semiconductor nanowires and its manipulation is, however, a complex task attracting a lot of attention [1]. It was demonstrated that nanowires exhibit a preferential growth direction (usually <111>) irrespective of the substrate crystallographic orientation. Schmidt et al. analyzed the nanowire growth considering surface free energies of the growth interface and nanowire sidewalls [2]. This concept based solely on thermodynamics introduced two possibilities to control the nanowire growth direction; either by catalyst engineering (thus potentially changing the solid-liquid interface energy) or by passivating nanowire sidewalls with different adsorbates (solid-vapor interface energy). Another success of Schmidt's hypothesis is in explaining an intriguing experimental observation that small diameter nanowires (usually $\langle 20 \mathrm{~nm}$ ) prefer to grow in $\langle 110\rangle$ directions, while the larger diameter ones grow in $\langle 111\rangle$ directions. This has been observed in many material systems grown from vapor phase molecular precursors, including $\mathrm{Si}$, Ge, III-Vs and II-VIs nanowires.

However, this hypothesis seems to be in contrast to experiments on Ge nanowires grown from atomic vapor (by evaporation from a solid source in MBE), which tend to grow in $\langle 110\rangle$ growth directions for a wide range of diameters [3]. Here, by utilizing atomic vapor Ge nanowires growth inside the SEM chamber on high-vacuum compatible sample heating stage with assistance of atomic hydrogen produced by dissociation of molecular hydrogen on heated tungsten wire, we are able to monitor in situ the process of the controlled nanowires growth. This method allowed achieving the same results as in deposition from the gas phase in terms of nanowire morphology and growth direction. In agreement with theoretical modeling [4] we show a real-time microscopic evidence of nanowire pedestal formation and associated droplet behavior, which determines the nanowire growth direction. Additionally, utilizing Auger Electron Microscopy we provide spectroscopic evidence that the adsorbed hydrogen prevents the gold diffusion from the nanowire tip to the sidewalls. Our successful attempts to intentionally kink the nanowires by introducing or pumping out the atomic hydrogen clearly demonstrate that thermodynamics is not the solely factor determining the nanowire growth direction and that there are energy barriers to overcome, mostly related to the droplet pinning on a nanowire tip [5].

References:

[1] S A Fortuna and X Li, Semicond. Sci. Technol. 25 (2010) 024005.

[2] V Schmidt, S. Senz, U Gösele, Nano Letters 5 (2005) 931.

[3] M Kolíbal et al. Applied Physics Letters 99 (2011) 143113. 
[4] K W Schwartz, J Tersoff, Nano Letters 11 (2011) 316.

[5] This research has been financially supported by the Ministry of Education, Youth and Sports of the Czech Republic under the project CEITEC 2020 (LQ1601) and by Grant Agency of the Czech Republic (16-16423Y). MK and TP acknowledge the support from FEI Company.

a)

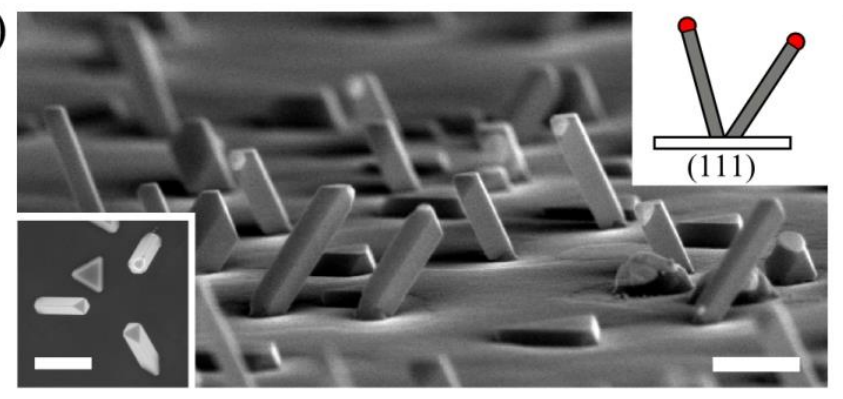

b)

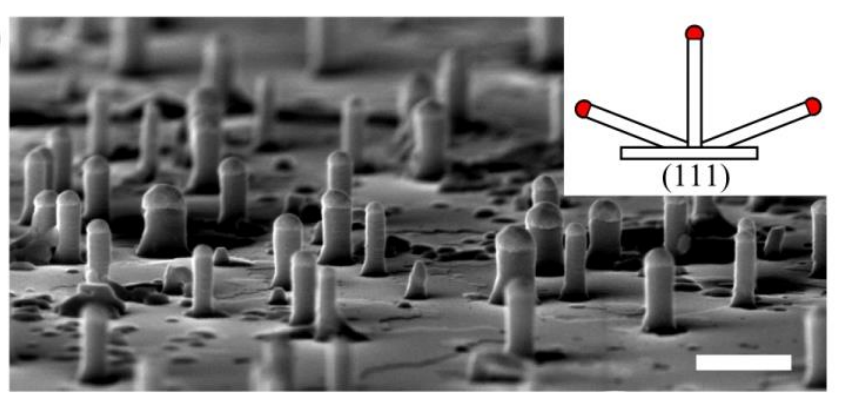

Figure 1. Germanium nanowires grown on the Ge(111) substrate under vacuum conditions (a) and at the presence of atomic hydrogen (b). The growth direction changes from $\langle 110\rangle$ to $\langle 111\rangle$. The inset in a) is a top view, the scale bars are $400 \mathrm{~nm}$.

(a)
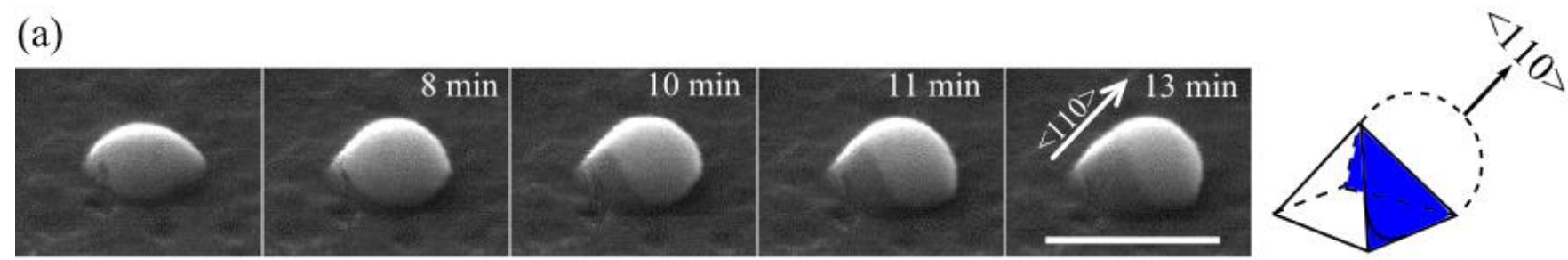

(b)
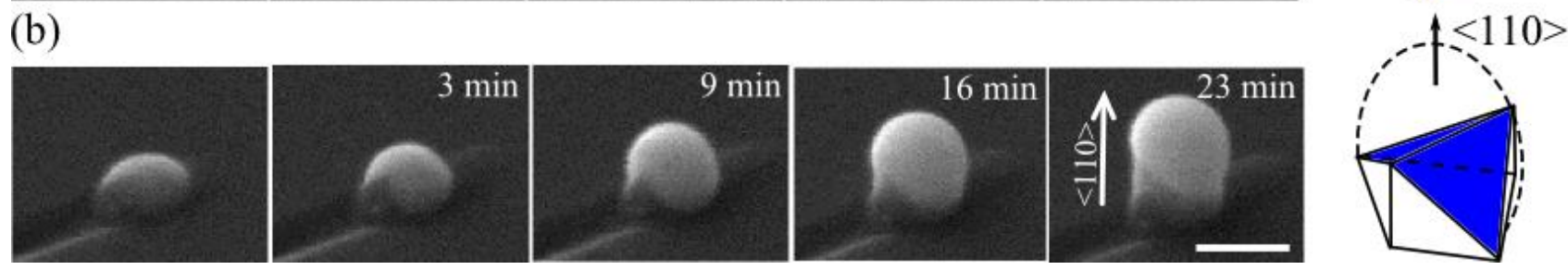

Figure 2. The formation of the V-shaped growth interface, possessed by nanowires growing in $\langle 110\rangle$ direction from different substrates under vacuum conditions (no hydrogen). a) An image sequence captured using scanning electron microscope at elevated temperature $\left(400{ }^{\circ} \mathrm{C}\right)$ during Ge evaporation (evaporation rate $3 \AA / \mathrm{min}$ ) onto $\mathrm{Ge}(100)$ substrate covered with Au nanoparticles (bright droplet in the middle of the image). b) Another experiment performed at higher evaporation rate (12 $\AA / \mathrm{min})$ on $\mathrm{Ge}(110)$ substrate. Again, the V-shaped interface is formed and in this case the nanowire grows perpendicular to the substrate. Scale bars, $200 \mathrm{~nm}$. The images are tilted by $52^{\circ}$ to the surface normal. 